\title{
Los centros de rehabilitación laboral para personas con enfermedad mental de la Comunidad de Madrid: un recurso para el empleo en tiempos de crisis (2008-2012)* Workplace rehabilitation centers for people with mental illness in Madrid: A resource for employment in crisis times (2008-2012)
}

\author{
Segundo Valmorisco Pizarro \\ Departamento de Análisis Social, Universidad Carlos III, Madrid, España. \\ segundo.valmorisco@uc3m.es
}

Resumen

En el presente artículo trata de identificar las variables que explican por qué los Centros de Rehabilitación Laboral (CRL) para personas con enfermedad mental grave y duradera de la Comunidad de Madrid consiguen tasas de inserción sociolaboral cercanas al 50\%, siendo un colectivo que presenta unas tasas de desempleo superiores al $80 \%$. Para ello se ha utilizado una metodología, en primer lugar, documental a través del uso de memoras de de actividad de los CRL de la Comunidad de Madrid durante el periodo 2008-2012. Y, en segundo lugar, cualitativa mediante entrevistas en profundidad a profesionales de diferentes perfiles de varios CRL, así como al Coordinador técnico de la red pública de atención social y a personas con enfermedad mental grave y duradera de la Comunidad de Madrid; y grupos de discusión a profesionales según categoría, así como a personas atendidas y familiares. Los resultados obtenidos muestran que la Red pública de atención a personas con enfermedad mental grave y duradera de la Comunidad de Madrid ofrece más de 5.900 plazas en diferentes recursos para el colectivo (centros de rehabilitación psicosocial, centros de día de soporte social, centros de rehabilitación laboral, residencias o pisos supervisados). En concreto, los CRL atienden a un total de 1.313 personas, de las cuales el $47,4 \%$ encuentran empleo (622 personas con enfermedad mental grave y duradera).

Palabras clave: equipo multidisciplinar, inserción sociolaboral, itinerario individualizado, rehabilitación laboral, trabajo en red.

\section{Abstract}

The current article tries to detect the variables that explain labour insertion rates (close to 50\%) of people with severe and enduring mental illness who come to work rehabilitation centres (CRL's) in the Community of Madrid. To this end, firstly, has been used a documentary methodology through the use of activity memoirs of the CRL's in the Community of Madrid with activity in 2008-2012. And, second, a qualitative methodology using In-depth interviews with professionals of different profiles of various CRL's as well as the technical coordinator of the public network of social care and people with severe and enduring mental illness of the Community of Madrid; and Focus groups according to professional category, as well as people served and family. The results show that the public network of care for people with severe and enduring mental illness, offers more than 5,900 seats in different collective resources (psychosocial rehabilitation centres, day centres, social support, vocational rehabilitation centres, nursing homes or supervised apartments). Specifically, CRL's serving a total of 1,313 people, of which $47.4 \%$ find employment (622 people with severe and enduring mental illness).

Key words: Individualized Itinerary, Multidisciplinary Team, Networking, Occupational Rehabilitation, Social and Professional Integration.

Sumario

1. Introducción | 1.1. Las personas con discapacidad por enfermedad mental| 1.2. Itinerarios de inserción sociolaboral|2. ¿Qué son los centros de rehabilitación laboral? | 3. ¿Por qué apenas existen evaluaciones de impacto de las políticas de empleo en España? | 4. Perfiltipo de la población atendida | 5. Algunos datos comparativos con otros recursos | 6. Factores de éxito de los CRL | 7. Conclusiones, propuestas y recomendaciones | Referencias bibliográficas

Cómo citar este artículo

Valmorisco Pizarro, S. (2015): “Los centros de rehabilitación laboral para personas con enfermedad mental de la Comunidad de Madrid: un recurso para el empleo en tiempos de crisis (2008-2012)", methaodos.revista de ciencias sociales, 3 (1): 7-18. http://dx.doi.org/10.17502/m.rcs.v3i1.64

\footnotetext{
* Esta investigación es parte de la tesis doctoral que el autor presentó en la Universidad Carlos III de Madrid.
} 


\section{Introducción}

El desempleo es un síntoma del anormal comportamiento de nuestro mercado de trabajo. Por desgracia, nos estamos acostumbrando a ver tasas de desempleo superiores al $20 \%$ que tienen su causa en diversos factores no corregidos a lo largo del tiempo en España. En el caso de las personas jóvenes y mayores de 45 años, estas tasas suponen varias veces la media de la UE.

Revisando los datos de la Encuesta de Población Activa (EPA) del Instituto Nacional de Estadística (INE), difícilmente podíamos imaginar en el segundo trimestre de 2007 que siete años después nuestra tasa de desempleo afectaría a 5,93 millones de personas, quienes representan el 25,93\% de la población activa (datos correspondientes al primer trimestre de 2014). En aquellos momentos, nos situábamos en una cifra más de tres veces inferior a la actual, con un histórico 7,93\% de paro que afectaba tan sólo a 1,77 millones de personas, por detrás de países como Francia y Alemania y tan sólo a un punto porcentual de la media de la UE. En aquel momento trabajaban en España 20,58 millones de personas, hoy en día a duras penas alcanzamos los 17 millones.

¿Qué hemos hecho mal en estos años para que nuestro mercado de trabajo arroje el peor comportamiento, junto a Grecia, de toda la Unión Europea? No es propósito de este artículo esgrimir las causas de semejante desastre, sino más bien identificar posibles soluciones a la situación actual a partir de la investigación cualitativa y documental de un recurso de empleo, que obtiene excelentes resultados de inserción sociolaboral: los Centros de Rehabilitación Laboral (CRL) para personas con enfermedad mental grave de la Comunidad de Madrid.

Para comenzar diremos que el recurso que se cita en este artículo existe doce años antes de que esta crisis económica y financiera comenzase a afectarnos de manera directa. Nos remontamos a 1996, año en que algunos técnicos de la Comunidad de Madrid se plantean alternativas al internamiento psiquiátrico de miles de personas, ofreciendo herramientas de inserción socio-laboral que complementasen los tratamientos de salud mental y los psicofármacos.

Hemos comenzado este artículo haciendo alusión a nuestra actual tasa de paro (como he indicado, ha llegado a estar muy próxima al 26\%), un porcentaje que supone un indicador globalizado para el conjunto de la población activa española mayor de 16 años. ¿Qué pasaría si esta referencia ascendiese hasta el $70 \%$ e incluso al $80 \%$ de total de una población específica? Efectivamente, el desempleo afecta a entre un $70 \%$ y un $80 \%$ de las personas con enfermedad mental grave y duradera de nuestro país, y si analizamos las alternativas ofrecidas por las diferentes Comunidades Autónomas, podemos comprobar como la oferta de recursos de empleo para este colectivo es muy diferente, y en la mayoría de las Comunidades Autónomas escasa y sin resultados. Después de un análisis documental exhaustivo es posible afirmar que la Comunidad de Madrid con sus CRL es una de las CC.AA que está a la cabeza en la gestión de itinerarios personalizados de inserción socio-laboral que desembocan en la creación de empleo para estas personas de tan difícil inserción, ya que, como habíamos indicado, según datos de la Red pública de atención social a personas con enfermedad mental de la Comunidad de Madrid, el 47,4\% acaban encontrando un empleo.

Tal y como se expresa en el título de este artículo, el objeto de estudio de esta investigación ha sido la inserción laboral de las personas con discapacidad por enfermedad mental a través de los itinerarios, aplicados al caso de los Centros de Rehabilitación Laboral (CRL) en nuestro ámbito de estudio: la Comunidad de Madrid, lugar donde se encuentra este singular recurso.

Para abordar esta triple realidad: itinerarios de inserción, personas con enfermedad mental y recursos o estructuras, como son los Centros de Rehabilitación Laboral donde se desarrollan estos itinerarios para esas personas, vamos a comenzar por entender cada una de estas tres dimensiones. Para ello, es necesario no sólo encuadrar unas ideas introductorias, sino también es importante cuantificar el número de personas a quienes afecta esta enfermedad y el número de centros y características básicas de los mismos.

\subsection{Las personas con discapacidad por enfermedad mental}

Para comenzar, debemos estimar la dimensión de nuestro objeto de estudio. Para ello hemos consultado diferentes fuentes que ofrecen información sobre la discapacidad en general y sobre la enfermedad mental 
en particular. Según la Encuesta de Discapacidad, Autonomía Personal y Situaciones de Dependencia (2008) -EDAD- elaborada por el INE, en España hay 3.847 .900 personas con discapacidad (el 8,24\% del total de la población española). De ellos, casi 1,5 millones tienen entre 16 y 64 años y 2,2 millones tienen 65 o más años. Del total de personas con discapacidad, 1,54 millones son varones y 2,30 millones son mujeres. En un total de 3,3 millones de hogares reside al menos una persona que afirma tener una discapacidad, lo que representa un $20 \%$ de los hogares españoles. De los mismos, 608.000 son hogares unipersonales, es decir, una persona con discapacidad que vive sola. Según el Centro de Investigación Biomédica en red de Salud Mental (Ministerio de ciencia e innovación del Gobierno de España e Instituto de Salud Carlos III), en España se considera que el 9\% de la población padece en la actualidad algún tipo de trastorno relacionado con la salud mental.

Para el Estudio ESEMeD-España 2006 (dicho estudio es parte de un proyecto europeo sobre epidemiología de los trastornos mentales, enmarcado dentro de una iniciativa de la World Mental Healt Surveys de la Organización Mundial de la Salud, llamada Encuestas de Salud Mental en el mundo) el 19,5\% de las personas presentaron un trastorno mental en algún momento de su vida (prevalencia-vida) y un 8,4\% en los últimos 12 meses (prevalencia-año). El trastorno mental más frecuente es el episodio depresivo mayor, que tiene un 3,9\% de prevalencia-año y un 10,5\% de prevalencia-vida. Después del episodio depresivo mayor, los trastornos con mayor prevalencia-vida son la fobia específica, el trastorno por abuso de alcohol y la distimia. Los factores asociados a padecer un trastorno mental según perfiles son el sexo femenino, estar separado, divorciado o viudo, estar desempleado, de baja laboral o con una incapacidad. Se puede estimar según los últimos datos que las enfermedades mentales consumen hasta el $20 \%$ del gasto sanitario en los países desarrollados, con una previsión al alza en los próximos años (XVII Simposio de la Fundación Lilly, 2010).

Un estudio reciente publicado en 2009 sobre el impacto socioeconómico de las enfermedades mentales en España (Moreno et al., 2009: 361-369) muestra las siguientes tendencias:

- Este tipo de enfermedades mentales son la segunda causa de baja temporal y permanente en el trabajo, después de las enfermedades osteomusculares.

- Además, las enfermedades mentales son la cuarta causa de cuidados informales (prestados por personas que no son profesionales, en general familiares), después de los accidentes cerebrovasculares, las demencias y las enfermedades osteomusculares.

- A pesar de los importantes recursos económicos y humanos destinados a la prevención y tratamiento de enfermedades mentales (visibles en las cifras del gasto sanitario estimado), el coste social "oculto" (pérdidas laborales y costes de cuidados informales) supone una carga social aún mayor.

- Según la investigación, los costes directos ocasionados por enfermedades mentales representan el $57,3 \%$ del total del coste estimado y el $42,7 \%$ restante representa la pérdida de productividad del trabajo.

- En referencia a los costes médicos directos, los gastos más significativos son las hospitalizaciones (19,1\% del coste total estimado) y los medicamentos (15,6\% del coste total estimado). En conjunto, los costes totales representaron en 2002 cerca del 1\% del producto interior bruto (PIB) de la economía española. Aunque los trastornos más frecuentes son depresión y ansiedad, los que generan más carga económica son la esquizofrenia y demencia.

Es muy importante tener en cuenta que la disponibilidad de las personas hacia el empleo, así como otras variables añadidas: autonomía, independencia o, entre otras, emancipación familiar es muy diferente según sean los siguientes rasgos.

- Género. Las mujeres están sujetas a una doble exclusión en el caso de la discapacidad. "En términos relativos, el 17,3\% de las mujeres con discapacidad se encontraba en situación de pobreza extrema, tasa que triplicaba la de la población española (6,4\%) en 2008 y era 
sensiblemente superior a la de los varones con discapacidad (11\%). El riesgo de pobreza no presenta diferencias significativas por género entre personas con discapacidad, aunque es prácticamente el doble para este grupo poblacional que para el resto de la población (51\% frente a 26\%)" (Huete, 2013: 29).

- Edad. La generación de los mayores de 45 años, quienes tenían 16 y más años en 1982, fecha de aprobación de la LISMI.

- Nivel académico. Generalmente, como ocurre con el mercado de trabajo en general, a más nivel académico mayor tasa de empleo. Según la Encuesta de Discapacidad, Autonomía Personal y Situaciones de Dependencia (EDAD) elaborada por el INE, de los casi 3,9 millones de personas con discapacidad, 198.600 tienen estudios universitarios o equivalente finalizados, esto es, sólo un $5,16 \%$ de la población con discapacidad alcanza el nivel educativo superior. Si se analiza la población entre 6 y 64 años el porcentaje alcanza el 7,88\%, siendo sólo el 0,84\% en la población entre 6 y 24 años. Según esta misma fuente, el 83,83\% de la población femenina con deficiencia mental (suma del conjunto de personas con enfermedad mental más las personas con discapacidad intelectual) posee estudios inferiores a educación secundaria, siendo en el caso de los varones del $72,67 \%$.

- Actitud y cultura hacia el empleo, variable en la que juega un papel fundamental la familia y el entorno.

- Tipo y grado de discapacidad, variable que puede limitar en mayor medida que el resto la capacidad de la persona para ocupar un puesto de trabajo con unas mínimas garantías.

\subsection{Itinerarios de inserción sociolaboral}

Al hablar de itinerarios de inserción socio-laboral, no existe un claro consenso en relación a las fases que contienen dicho itinerario, tanto desde el ámbito de los expertos como de algunas de las entidades sociales más especializadas (por ejemplo, Fundación Tomillo y Fundació Pere Tarrés). En este sentido, podría decirse que todos los servicios de empleo, tanto públicos como privados: ONG, Sindicatos, Patronal, Administración Pública y Universidades, hacen uso de su propio itinerario, adaptando en cada caso, y en ocasiones sin un modelo teórico claro, las diferentes fases a cada persona, sin que podamos hablar de un protocolo cerrado ni consensuado para ello. Podemos considerar, que estos seudo-modelos adolecen en muchos casos de deficiencias metodológicas que posiblemente afecten a su resultado final.

Figura 1. Itinerario aplicado en los Centros de Rehabilitación Laboral (CRL).

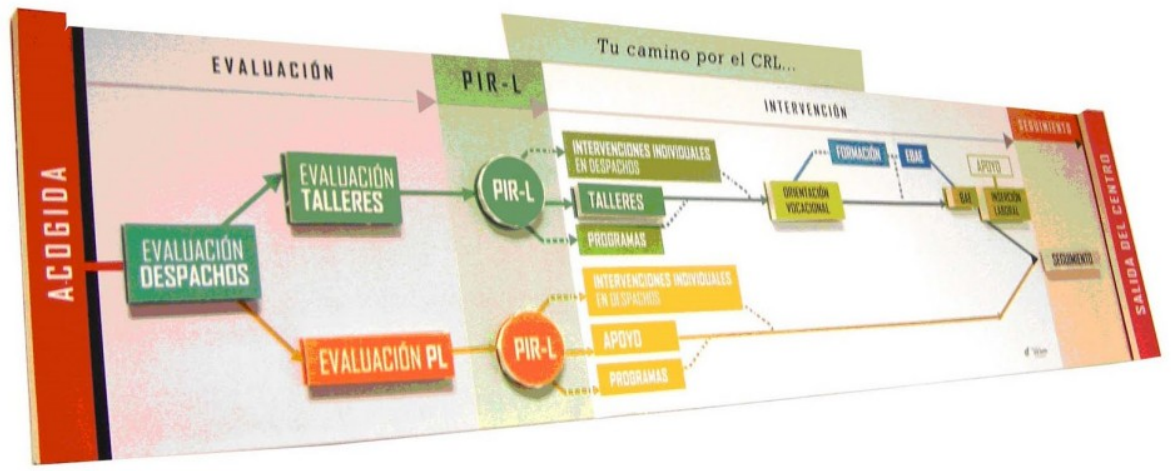

Fuente: Dirección General de Servicios Sociales, Comunidad Autónoma de Madrid. 
El modelo lineal aplicado por los CRL presenta la novedad de incorporar la evaluación nada más terminar la fase de acogida, no así al terminar cada una de las diferentes fases del total del proceso (como ocurre en el modelo de la Fundación Tomillo). Esta fase de evaluación se realiza por cada uno de los profesionales que componen el equipo de trabajo (psicólogo, TAIL, preparador laboral, terapeuta ocupacional y maestros de taller), así como se evalúa el comportamiento de cada persona en los talleres pre-laborales durante su estancia previa de 45 días. Es necesario apuntar un par de cuestiones: a) anualmente se realiza una evaluación de los objetivos con la persona atendida y estos pueden cambiar en función de la misma y b) este itinerario no es lineal, sino que ofrece en la práctica bastante flexibilidad, de modo que una persona puede pasar del PIR-L a la fase de formación o directamente al empleo si así lo aconsejan las circunstancias.

Si bien existen en el modelo dos puntos de evaluación del proceso: una evaluación que se hace manifiesta en los primeros 45 días de la persona en el recurso y otra al final del itinerario, consideramos que un exceso de evaluación no garantiza el éxito de la inserción laboral, como tampoco es crucial evaluar al final de un proceso tan largo, así como no contar con el feed-back de la información por parte de la persona. Una vez terminado este primer proceso evaluador, posterior a la fase de acogida, se convoca una Junta de Evaluación, en la que participan todos los profesionales del CRL que han analizado la inmersión de la persona en los diferentes talleres. Con toda esa información se establece y propone el Plan Individualizado de Recuperación Laboral (PIRL). Este PIRL se remite a Salud Mental, organismo de quien se recibe la derivación de la persona.

En definitiva, las fases de forma resumida son: acogida, evaluación, PIRL, intervención, seguimiento y alta, siendo la segunda fase la que presenta diferencias con cualquier otro modelo estudiado. Como podemos comprobar en la Figura 1 existen dos rutas bien diferenciadas: a) la ruta superior se lleva a cabo entre las personas que acuden al CRL y no tienen empleo y b) la ruta inferior se lleva a cabo entre las personas que acuden al recurso con un puesto de trabajo y precisan de herramientas para mantenerlo. Este segundo grupo tienen como profesional de referencia al preparador laboral, quien realiza tareas de Empleo con apoyo. Las personas atendidas que se encuentran en este itinerario no acuden a los talleres, pero se les hace un PIR-L y se les ofrece apoyo, así como los programas propios de cada centro. El itinerario para este segunda ruta sigue con las fases de seguimiento (puesto que la persona ya tiene un empleo) y alta.

En la ruta superior, una vez terminado el periodo de 45 días que culmina con la elaboración compartida junto a la persona de su Plan Individualizado de Rehabilitación Laboral (PIR-L), plan en el que se marcan sus objetivos a conseguir, comienza la fase de intervención en la que la persona se incorpora de forma definitiva a los talleres y programas del centro, así como se continúan con las intervenciones individuales en despachos con cada uno de los profesionales. Esta fase de intervención abarca: trabajo en programas y talleres (así como intervenciones individuales en despachos), Orientación vocacional, formación, Entrenamiento en Búsqueda Activa de Empleo (EBAE), BAE, Inserción laboral y empleo con apoyo.

Otra característica importante de este modelo es que la fase de orientación vocacional se encuentra ya en el propio proceso de intervención, y no antes, como así ocurre en la mayoría de modelos. De esta forma se asesora para "pulir" mejor la identificación de capacidades e intereses laborales de la persona. Esta fase es anterior a la fase de formación y de búsqueda activa de empleo (BAE) y a la fase de intermediación laboral, es decir, la persona atendida ya participa de los diferentes talleres y aprende haciendo. En esta fase de orientación vocacional, el profesional de referencia es el terapeuta ocupacional.

El siguiente proceso en la fase de intervención, es el de identificar las necesidades de formación de la persona, formación que generalmente se realiza fuera del propio centro. A partir de este proceso la persona se centra en el empleo, comenzando con el Entrenamiento en Búsqueda Activa de Empleo y con la propia BAE. Posteriormente le sigue la fase de Inserción laboral con empleo con apoyo. En ambos casos (ruta superior y ruta inferior) el itinerario termina con la fase de seguimiento de la persona insertada y con la fase de alta.

Debemos considerar la importancia de los itinerarios de creación de empleo para cualquier persona que esté buscando empleo. Dependiendo de los perfiles profesionales es posible obviar alguna de sus fases, sin embargo, dadas las especiales características de las personas con enfermedad mental, no es conveniente modificar el esquema-modelo del mismo (y mucho menos eliminar alguna de sus fases). En este sentido la fase de orientación laboral-vocacional no debe realizarse únicamente sobre la base de lo 
que el sujeto nos trasmite como preferencias de ocupación. Hemos de dedicar el tiempo necesario, y más con quien padece una enfermedad mental, para ayudarle a organizar y reorientar sus preferencias desde un punto de vista objetivo, aparte de sus preferencias y experiencia laboral previa.

Uno de los factores de éxito de este recurso es poder comprobar in situ como trabaja la persona y se desenvuelve en los diferentes programas y talleres en los que de forma consensuada ha entrado desde la aceptación bilateral del PIRL. Este extremo apenas es comparable en otros recursos, si acaso en los programas mixtos de empleo y formación con amplio recorrido en España, como son las escuelas taller, las casas de oficios y los talleres de empleo.

\section{2. ¿Qué son los centros de rehabilitación laboral?}

Los CRL (como hemos indicado, fundados en 1996 por la Comunidad de Madrid), constituyen el recurso social específico (dependientes del Programa de Atención Social a Personas con Enfermedad Mental Crónica), cuya misión fundamental es favorecer la rehabilitación vocacional-laboral de las personas con trastornos psiquiátricos crónicos que se encuentran viviendo en la comunidad, de modo que propicie su integración laboral normalizada fundamentalmente en la empresa ordinaria o también en fórmulas de empleo protegido (Centros Especiales de Empleo) o sistemas de auto-empleo. Los CRL no tienen carácter de centro de trabajo, por lo que no pueden participar en operaciones regulares de mercado ni incluir relaciones laborales remuneradas con sus usuarios, como si lo hacen otros recursos tales como los Centros Especiales de Empleo o las Empresas de Inserción, donde si es posible establecer relaciones de trabajo con las personas que pertenecen a dichos centros, así como entre estas entidades "empresariales" y otras empresas para la prestación de bienes o servicios.

En la actualidad existen en la Comunidad de Madrid 21 CRL. Todos ellos están compuestos por un equipo multiprofesional formado por: 1 Director (psicólogo), 1 Psicólogo, 1 Terapeuta Ocupacional, 1 Técnico de Apoyo a la Integración Laboral, 1 Preparador Laboral, 3 Maestros de Taller, 1 Auxiliar Administrativo, $1 \frac{1}{2}$ Auxiliar de Limpieza.

Junto a los resultados de inserción y a la composición de los equipos de trabajo de los CRL, hemos de considerar la estabilidad financiera del recurso, ya que en tiempos de recortes presupuestarios, apenas ha sufrido ajustes: incrementándose un 15,5\% entre 2008 y 2012, pasando de 56 a 64,7 millones de euros anuales. Según los últimos datos disponibles, y habiéndose recortado el presupuesto anual de 2013 en un $1,70 \%$ respecto a 2012 , se ha producido un ligero aumento en el número de plazas.

\section{3. ¿Por qué apenas existen evaluaciones de impacto de las políticas de empleo en España?}

Para que las políticas de empleo sean efectivas, éstas deberían centrarse en crear puestos de trabajo para las personas. Esta afirmación que parece tan simple, no lo es tanto si analizáramos los resultados de las políticas activas de empleo en España. Y digo si analizáramos porque no existen evaluaciones rigurosas de esas políticas para conocer el estado de la cuestión. Podríamos confirmar la prácticamente nula existencia de informes que hayan analizado el impacto y la relevancia de las políticas de empleo en la creación de puestos de trabajo. Si bien es cierto que los destinatarios de estas políticas han sido en muchos casos colectivos desfavorecidos, tales como: personas con discapacidad, jóvenes menores de 25 años, personas mayores de 45 años, mujeres e inmigrantes, todos ellos siempre han presentado unas tasas de desempleo de partida inferiores a las personas con enfermedad mental grave.

Los CRL corrigen esta deficiencia, en cuanto a la inexistencia de evaluaciones, realizando análisis anuales en relación al número de personas insertadas y las características de dicha inserción. De esta forma, se identifican los factores de éxito, así como las debilidades a corregir.

\section{Perfil-tipo de la población atendida}

El colectivo de personas con enfermedad mental grave presenta diferencias en cuanto a las condiciones de partida de cada una de las personas atendidas. Dichas condiciones ejercen una influencia directa en sus 
resultados de inserción: tipo de enfermedad mental, ingresos económicos de los hogares, nivel de funcionamiento psicosocial, estado civil, experiencia laboral previa o nivel académico.

En concreto, el perfil-tipo de los usuarios/as que acuden a los CRL es el siguiente: todas las personas presentan enfermedad mental grave y duradera, siendo la esquizofrenia, otros trastornos psicóticos y los trastornos de personalidad los diagnósticos clínicos más frecuentes, en más de $70 \%$ de los casos tienen ingresos económicos propios procedentes de: su propio trabajo, pensión no contributiva o prestación por hijo a cargo. Casi el $90 \%$ son personas solteras con un nivel académico de Enseñanza primaria, EGB ( $2^{\mathrm{a}}$ Etapa: $8^{\circ}$ ) o Bachiller Superior, BUP, COU, Bachillerato y con alguna experiencia laboral previa.

Entonces cabe preguntarse: ¿es posible identificar factores de éxito en un recurso que posibilita que casi el $50 \%$ de las personas con enfermedad mental grave y duradera encuentren un empleo? Imaginemos por un momento que cualquier política de empleo consiguiese que la mitad de las personas atendidas encontrasen un trabajo. Algunos de esos factores de éxito han sido señalados anteriormente: existencia de un compensado equipo multiprofesional, financiación estable y su resultado de inserción socio-laboral $(47,4 \%)$.

\section{Algunos datos comparativos con otros recursos}

Tratando de dar respuesta al interrogante planteado en un epígrafe anterior y observando el estado de la cuestión en la actualidad, si hoy en día visitamos recursos municipales públicos, que desarrollen programas destinados a la búsqueda de empleo de personas desempleadas, veremos la importante reducción de personal y medios para esta importante tarea, cuando esta cifra en muchos municipios se ha triplicado. Gran parte de las tareas de intermediación laboral que en otro momento hicieron las Agencias Locales de Empleo, los $\mathrm{CIE}$, etc., hoy están en manos de las Agencias de Colocación. Debemos esperar algún tiempo para evaluar el funcionamiento de este nuevo recurso, que ha pasado a compartir misión con la iniciativa privada (las entidades autorizadas ahora son Empresas de Trabajo Temporal, Ayuntamientos, ONG, etc.).

Entonces, ¿qué diferencias presentan los CRL respecto a otros recursos o políticas de empleo desarrolladas en España? Los datos objetivos nos dicen que, por ejemplo, en el ámbito geográfico de la Comunidad de Madrid, las Empresas de Inserción sostienen en torno a unos 100 puestos de trabajo (en la actualidad existen 12 EI inscritas en el registro correspondiente en la Comunidad de Madrid, con una media de 7,8 puestos de trabajo creados). Otro recurso, como son los Centros Especiales de Empleo (con 179 centros registrados en la actualidad en la misma Comunidad Autónoma), se estima según los datos ofrecidos por la Federación Empresarial Española de Asociaciones de CEE (FEACEM) que pueden estar creando en torno a 5.500 puestos de trabajo en la Comunidad de Madrid (con unos 30 empleos de media por cada CEE).

De media, cada CRL (de los 21 existentes), sin ser un recurso finalista como son los CEE o las EI (ya que se dedica a la intermediación laboral y a preparar a los usuarios para el empleo), crea unos 30 puestos de trabajo anuales, es decir más de 4 veces más que las EI y al mismo nivel que los $C E E$, aun trabajando con colectivos de igual o más difícil inserción como son los colectivos atendidos por las EI y los propios CEE. La diferencia por tanto, radica en que no estamos hablando de un recurso cuya finalidad exclusiva sea la creación de empleo (como sí lo son los CEE, quienes se definen como fórmulas de empleo cuyo objetivo principal es la inserción laboral), sino favorecer la rehabilitación laboral, como paso previo a la inserción sociolaboral de la persona, de modo que aún sin ser su objetivo principal la creación de empleo es capaz de crear, de media, el mismo número de puestos de trabajo que otro recurso finalista como son los CEE.

\section{Factores de éxito de los CRL}

Las altas tasas de inserción sociolaboral de los CRL de la Comunidad de Madrid son producto de la conjunción de varios factores: la ubicación del recurso en el ámbito de los servicios sociales (si bien debería establecerse una mayor conexión con el área de empleo); el liderazgo de los técnicos de alto nivel y su coordinación con las direcciones generales y consejerías implicadas; la alta competencia, vocación y 
motivación de los equipos profesionales; así como la fórmula de financiación a través del concierto (con unos pliegos que especifican al detalle las condiciones técnicas).

El éxito de este modelo de gestión, se explica por todos estos factores y especialmente en el análisis comparativo de los diferentes recursos en las Comunidades Autónomas por: a) la coordinación con salud mental en la derivación de casos; b) la gestión compartida con la persona de las diferentes fases del itinerario; c) la armonizada composición del equipo de trabajo (especialmente con la figura del Técnico de Apoyo a la Inserción Laboral); d) la apuesta de la Comunidad de Madrid al mantener el presupuesto; y e) el liderazgo de los técnicos de alto nivel y su coordinación con las direcciones generales y consejerías implicadas, son algunos de los factores que explican el éxito del modelo.

Desde el recurso de los CRL se ha comprobado que los factores de éxito para la inserción laboral de las personas usuarias son: a) motivación de la persona; b) el apoyo familiar recibido; c) las experiencias laborales previas; y d) el buen nivel de funcionamiento (habilidades y capacidades). Todos estos aspectos son trabajados en los CRL, a excepción de las experiencias laborales previas. No obstante, se pulen las competencias adquiridas en el pasado y se adaptan las mismas, así como se adquieren otras nuevas a través de los diferentes programas en los que la persona participa.

Tal y como hemos podido comprobar en la investigación cualitativa realizada, algunos de estos mismos agentes podrían inhibir la inserción sociolaboral de las personas atendidas, a saber: no sólo la propia persona y la familia, sino también la sociedad y el propio equipo profesional. De modo que podríamos situar cuatro agentes claros en el proceso de rehabilitación laboral (Tabla 1).

Tabla 1. Agentes clave en el proceso de rehabilitación laboral.

\begin{tabular}{cc}
\hline Persona & Familia \\
\hline $\begin{array}{c}\text { Sociedad-Políticas } \\
\text { (Micro y Macro) }\end{array}$ & $\begin{array}{c}\text { Equipos profesionales } \\
\text { (Salud mental y CRL) }\end{array}$ \\
\hline Fuente: elaboración propia.
\end{tabular}

Por otro lado, como ya hemos indicado, otro factor de éxito tiene que ver con que la crisis no ha afectado a la estabilidad del recurso, como lo ha hecho en otras Comunidades Autónomas, en la medida en que este modelo ha estado protegido tanto en efectivos como en dotación presupuestaria por la propia Comunidad de Madrid. Bien es cierto que la Red de Atención Social a personas con enfermedad mental ha sufrido un pequeño ajuste presupuestario, por ejemplo en el periodo 2012-2013 el presupuesto se redujo un 1,70\%, pasando de los 64,7 millones en 2012 a los 63,6 millones en 2013. No obstante, hemos de decir que a pesar de este pequeño ajuste y de una coyuntura desfavorable, el número de plazas se ha incrementado en el periodo estudiado, e incluso en la actualidad sigue esta tendencia al aumentar en 55 plazas nuevas para el año 2014 (hasta llegar a las 1.035 existentes en la actualidad).

\section{Conclusiones, propuestas y recomendaciones}

Una vez expuestas las principales características del recurso, vamos a enumerar cuales son las conclusiones obtenidas sobre el mismo, separando entre aquellas conclusiones obtenidas a partir del análisis específico del recurso en la Comunidad de Madrid, de las obtenidas en comparación con otras CC.AA. Respecto a la Comunidad de Madrid:

- Este recurso crea un subsistema propio de empleo. La generación de empleo protegido fomentado por los mismos asciende al $47 \%$ del total de empleos generados (porcentaje que sube hasta el 52\% al cierre de 2013), si este recurso desaparece, se deja de prestar atención a miles de personas.

- Vital importancia del Trabajo en red. Es difícil concebir la integración laboral de las personas con enfermedad mental usuarias de los CRL sin la presencia del trabajo en red que realizan con los CEE, sobre todo en el caso del País Vasco y Andalucía, y de forma incipiente también ahora en la Comunidad de Madrid. De hecho, según los datos del Observatorio Regional de empleo de la Comunidad de Madrid sobre contratación registrada en personas con discapacidad a nivel global 
en 2012 (no exclusivamente de personas con enfermedad mental) indican que casi el $70 \%$ del total de contratos proceden de los CEE.

- Es posible considerar la importancia de los itinerarios de creación de empleo para cualquier persona que esté buscando empleo. Como ya hemos señalado, dependiendo de los perfiles profesionales es posible obviar alguna de sus fases, sin embargo, dadas las especiales características de las personas con enfermedad mental, no es conveniente modificar el esquemamodelo del mismo, en este sentido la fase de orientación laboral-vocacional no debe realizarse únicamente sobre la base de lo que el sujeto nos trasmite como preferencias de ocupación, hemos de dedicar el tiempo necesario, y más con quien padece una enfermedad mental, para ayudarle a organizar y reorientar sus preferencias desde un punto de vista objetivo, aparte de sus preferencias y experiencia laboral previa.

- Se trata de un recurso que ofrece gran flexibilidad en la prospección de proyectos, lo que da una amplia cabida para la innovación dentro del propio recurso.

En relación a otras Comunidades Autónomas:

- Según el Observatorio Español de Rehabilitación Psicosocial (Federación Española de Asociaciones de Rehabilitación Psicosocial), solamente 5 Comunidades Autónomas ofrecen servicios de rehabilitación laboral. Estas son: Andalucía, Galicia, Cataluña, Comunidad de Madrid y Castilla- La Mancha.

- El periodo de crisis que nos afecta y que presumiblemente seguirá afectando a la creación de empleo en los próximos años, no ayuda en este sentido, por lo que se hace más necesario que nunca, la búsqueda de fórmulas que ayuden a los profesionales que diseñan estos itinerarios a basar la evaluación de competencias en datos objetivos observados, más que en simples entrevistas ocupacionales que contengan la información no contrastada de las personas a quienes atienden. En este sentido, las fases y los recursos de los propios itinerarios deben ser articulados en un mismo procedimiento y recogidos en un mismo espacio y, si esto no fuera posible, innovar en procedimientos (convenios, contratos, planes, etc.) que garanticen, para las personas en riesgo de exclusión social, el incremento de sus capacidades y nuevas oportunidades para su plena integración en el mercado de trabajo.

De las conclusiones expuestas es posible extraer una serie de consecuencias que nos animan a realizar las siguientes propuestas de mejora:

- Una primera conclusión extraída de este análisis comparativo del recurso en las diferentes Comunidades Autónomas, es la falta de liderazgo por parte de la Administración del Estado para coordinar unas competencias que, precisamente porque pertenecen a las Comunidades Autónomas, reproducen un auténtico galimatías, al establecerse 17 modelos diferentes de atención. Bien este cierto, que existen multitud de variables que interaccionan entre sí y en ocasiones impiden su buen desarrollo, y más en un país tan diverso como es España: distintas densidades de población, diferentes formas de concebir el modelo, atomización poblacional, etc., todo ello hace que cada Comunidades Autónomas actúe de forma propia.

- Recomendamos la creación de una gran base de datos que abarque a las personas atendidas en los diferentes dispositivos de rehabilitación laboral (en todas las fases del proceso), de modo que se identifiquen en la misma las variables objeto de un amplio estudio cuantitativo, a través del programa estadístico IBM SPSS o similares. Consideramos que por los resultados alcanzados en inserción laboral (recordemos que su tasa de inserción es probablemente la más alta de España) se debería liderar desde los CRL un gran estudio a nivel nacional que aporte evidencias empíricas e identifique los factores de éxito y recomiende en cada caso el itinerario y los modelos de rehabilitación laboral más convenientes. 
- Se recomienda un análisis en profundidad de las competencias que abarcan cada puesto de trabajo, para centrar la I+D de los CRL en la consecución de esas competencias mediante adaptaciones.

- A pesar de haber identificado con la investigación cualitativa y documental los factores de éxito de este recurso (aunque sin claras evidencias empíricas a nivel cuantitativo, más allá de las tasas de inserción laboral, producto de las memorias de actividad), debemos reconocer que es necesario un profundo análisis mediante la explotación de una segunda base de datos compuesta por personas atendidas que se encuentren únicamente en la fase de seguimiento, para poder llegar a cuantificar y ponderar la importancia de estas variables que influyen en la inserción sociolaboral de estas personas. Y esta recomendación debería estar incluida en una evaluación global del recurso.

- Se debería poder analizar cuál es el retorno de la inversión en rehabilitación laboral en cuanto a ahorro económico del Estado al impulsar este tipo de recursos.

- Convendría implementar procedimientos de diagnóstico precoz en los servicios de atención primaria: la media en diagnosticar una enfermedad mental es de 10 años.

- Se recomienda en el nivel nacional (no siendo el caso de los CRL de la Comunidad de Madrid) una financiación estable para evitar que los equipos profesionales fluctúen y no puedan garantizar la atención de las personas atendidas.

Dicho esto, en este trabajo se identifican algunos de los elementos que facilitan las buenas prácticas en este tipo de recursos y que arrojan importantes cifras de inserción laboral (no sólo en el empleo protegido, sino también en las empresas ordinarias) para ofrecer esos modelos de éxito a todas las Comunidades Autónomas para su puesta en funcionamiento. Obviamente y como acabamos de indicar, todo ello debe ser apoyado económicamente creando un presupuesto anual para tal fin, de esta forma se lograría equiparar y nivelar los diferentes servicios prestados por cada recurso. Pero todo esto no sería posible sin un amplio consenso y puesta en común de todas y cada una de las Administraciones regionales, un consenso al estilo del realizado en 1990 para la elaboración del Plan Concertado de Servicios Sociales.

Es posible y factible hacer esta propuesta en la medida en que existen algunos precedentes. En este sentido, la Comunidad de Madrid sí ha logrado importantes consensos para implantar este recurso multinivel, que abarca diversas áreas, como pueden ser: empleo, formación, servicios sociales y salud, dentro de la Administración regional, pero que también involucra a empresas y Asociaciones y Fundaciones, todas ellas persiguiendo un mismo fin, a sabiendas de los beneficios que produce, en este colectivo, la inserción en el mercado de trabajo.

Por todo ello, podemos decir que los CRL son un modelo de gestión de la inserción sociolaboral de colectivos con grave riesgo de exclusión social, y por tanto, podrían ofrecer una serie de recomendaciones a otros servicios de empleo. Estas son:

- Sería necesario una legislación de Servicios Sociales a nivel estatal que acogiera unos criterios mínimos de armonización para la rehabilitación laboral de las personas con discapacidad por enfermedad mental grave y duradera.

- Dado el contexto de actual crisis económica y financiera habría que apostar por favorecer la financiación para este tipo de recursos de forma que se acabe con los recortes en conciertos y subvenciones en la mayoría de Comunidades Autónomas.

- Impulsar una mayor coordinación entre los diferentes modelos en cada Comunidad Autónoma, a sabiendas de que somos mucho menos eficaces sin coordinación. De esta forma se podría protocolizar el modelo de atención. 
- Fomentar el liderazgo por parte de la Administración estatal para comenzar un proceso de unificación de criterios, financiación, equipos profesionales o infraestructuras. En ese liderazgo debe primar lo técnico frente a lo político.

- Trabajar contra: el estigma de la sociedad (a nivel macro). Haría falta una gran campaña de publicidad sobre la capacidad y la necesidad de empleo en las personas con enfermedad mental. También contra el estigma de la micro-sociedad: calle, barrio, etc., y el autoestigma de las propias personas con enfermedad mental.

- Promover una mayor participación de las familias en todo el proceso de rehabilitación laboral favoreciendo una mayor información y evitando la sobreprotección de las mismas hacia las personas con enfermedad mental.

- Realizar campañas para luchar contra la desinformación de los empresarios en este campo.

- Fomentar el Autoempleo: apenas se crean puestos de trabajo bajo esta modalidad. Requiere de unas mayores competencias. Los CRL reflejan una realidad social (escasez de autoempleo, aparte de las barreras existentes en España para el autoempleo: trato fiscal, burocracia, etc.).

- Trabajar en pos de una mayor coordinación con el resto de consejerías (en el caso de la Comunidad de Madrid, con el Servicio Regional de Empleo, la Consejería de Empleo y los Centros Base del IMSERSO).

- Ampliar la oferta de cursos de formación adaptados a nuestro colectivo.

- Establecer políticas de discriminación positiva hacia las mujeres.

- Apoyar en mayor medida a las familias, las cuales afirman estar poco preparadas para ayudarles.

- Promover el diagnóstico precoz en los servicios de atención primaria (la media en diagnosticar a una persona es de 10 años).

- Afianzar un recurso que consigue, probablemente, la más alta tasa de inserción laboral del país para las personas con enfermedad mental.

Consideramos que, de cumplirse las recomendaciones multinivel aquí expuestas, la rehabilitación laboral para personas con enfermedad mental grave y duradera en el ámbito nacional conseguiría mejores resultados de inserción y, con ello, se mejoraría la calidad de vida de este colectivo. Entendemos, que el estudio de caso de la Comunidad de Madrid analizado en esta investigación, aunque presenta algunos defectos que deberían ser corregidos, ofrece evidencias suficientes como para poder ser identificado como una buena práctica (consideramos que el volumen de casos con el que trabaja el recurso, así como el porcentaje de inserción laboral conseguida, son datos suficientes, para hacer esta afirmación), por lo que estamos ante un modelo de referencia que debe ser tomado en cuenta para planificar la rehabilitación laboral en España. Para ello es necesario iniciar de forma urgente una estrategia a nivel nacional (que tendrá repercusiones posteriores en las Comunidades Autónomas) en la que confluyan un firme liderazgo político y un sólido trabajo técnico en el que tengan cabida la Investigación y el Desarrollo.

\section{Referencias bibliográficas}

Alonso, L. E. y Pérez Ortiz, L. (1996): ¿Trabajo para todos?: un debate necesario. Madrid: Ediciones Encuentro. 
Asociación Española de Neuropsiquiatría (2002): Rehabilitación psicosocial del trastorno mental severo. Situación actual y recomendaciones. Madrid: Cuadernos Técnicos, 6 .

Bedia, M. y Arrieta, M. (2001): "Rehabilitación sociolaboral de personas con esquizofrenia: variables relacionadas con el proceso de inserción", Actas Españolas de Psiquiatría, 29 (6): 357-367.

Ceniceros, J. C. y Oteo, E. (2003): La Orientación sociolaboral basada en itinerarios. Una propuesta metodológica para la intervención con personas en riesgo de exclusión. Madrid: Fundación Tomillo.

Comunidad de Madrid (2001): Rehabilitación laboral de personas con enfermedad mental crónica: Programas básicos de intervención. Madrid: Cuadernos Técnicos de Servicios Sociales, 14.

Fondo Social Europeo y Junta de Andalucía (2007): Los programas de empleo para personas con trastornos mentales graves. Una versión actualizada de los modelos de intervención. Sevilla. Fondo Social Europeo y Junta de Andalucía

Guerrero, C. (2005): "Itinerarios de inserción sociolaboral: una propuesta metodológica para personas en riesgo de exclusión social", Revista REDSI - Red Social Interactiva, 6.

Haro. J. M. y Palacín, C. (2006): Prevalencia de los trastornos mentales y factores asociados: resultados del estudio ESEMeD-España. San Boi de Llobregat, Barcelona: Unidad de Investigación y Desarrollo, Sant Joan de Déu-Serveis de Salut Mental. http://dx.doi.org/10.1157/13086324

Huete, A. (2013): Pobreza y exclusión social de las mujeres con discapacidad en España. Madrid: Cinca.

INE (2008): Encuesta sobre Discapacidades, Autonomía Personal y Situaciones de Dependencia 2008. Madrid: Instituto Nacional de Estadística.

- (2014): El empleo de las personas con discapacidad (2008-2012). Madrid: Instituto Nacional de Estadística.

Moreno, J. O., López-Bastida, J., Montejo-González, A. L., Osuna-Guerrero, R. y Duque-González, B. (2009): "The socioeconomic costs of mental illness in Spain", European Journal of Health Economics, 10: 361 369. http://dx.doi.org/10.1007/s10198-008-0135-0

Observatorio de Rehabilitación Psicosocial (2012): Valoración por comunidades del impacto de la crisis económica en la atención de rehabilitación psicosocial RPS. Madrid: Federación Española de Asociaciones de Rehabilitación Psicosocial.

Pastor, A., Blanco, A. y Navarro, D. Coords. (2010): Manual del trastorno mental grave. Madrid: Síntesis.

Rodríguez, A. Coord. (1997): Rehabilitación psicosocial de personas con trastornos mentales crónicos. Madrid: Pirámide.

Rodríguez González, A. (2001): "La experiencia de los Centros de Rehabilitación Laboral de la Comunidad de Madrid", Revista de la Asociación Madrileña de Rehabilitación Psicosocial, 8 (13): 5-12.

Rodríguez, A. y González, J. C. (2002): La rehabilitación psicosocial en el marco de la atención comunitaria integral a la población enferma mental crónica. Madrid: Cuadernos técnicos de servicios sociales, 17.

\section{Breve CV del autor}

Segundo Valmorisco Pizarro es Diplomado en Trabajo Social por la Universidad Pontifica de Comillas, Licenciado en Sociología por la Universidad Pontificia de Salamanca y Doctor por la Universidad Carlos III de Madrid. En la actualidad es profesor asociado en la Universidad Complutense de Madrid y en la Universidad Carlos III de Madrid. Sus principales líneas de investigación son el análisis y evaluación del sistema español de formación continua y de la inserción sociolaboral de personas con discapacidad por enfermedad mental grave. 